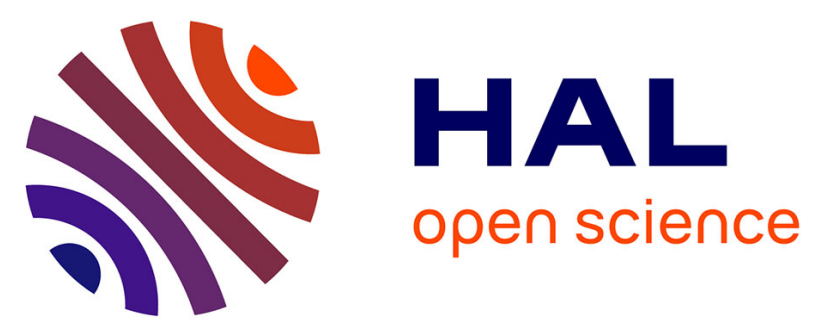

\title{
Exponential Stabilization of a Class of Nonlinear Neutral Type Time-Delay Systems, an Oilwell Drilling Model Example
}

\author{
Martha Belem Saldivar, Alexandre Seuret, Sabine Mondié
}

\section{To cite this version:}

Martha Belem Saldivar, Alexandre Seuret, Sabine Mondié. Exponential Stabilization of a Class of Nonlinear Neutral Type Time-Delay Systems, an Oilwell Drilling Model Example. CCE 2011 - 8th International Conference on Electrical Engineering, Computing Science and Automatic Control, Oct 2011, Mérida City, Yucatan, Mexico. pp.n.c. hal-00632606

\section{HAL Id: hal-00632606 https://hal.science/hal-00632606}

Submitted on 14 Oct 2011

HAL is a multi-disciplinary open access archive for the deposit and dissemination of scientific research documents, whether they are published or not. The documents may come from teaching and research institutions in France or abroad, or from public or private research centers.
L'archive ouverte pluridisciplinaire HAL, est destinée au dépôt et à la diffusion de documents scientifiques de niveau recherche, publiés ou non, émanant des établissements d'enseignement et de recherche français ou étrangers, des laboratoires publics ou privés. 


\title{
Exponential Stabilization of a Class of Nonlinear Neutral Type Time-Delay Systems, an Oilwell Drilling Model Example*
}

\author{
Martha Belem Saldivar ${ }^{1,2}$, Alexandre Seuret ${ }^{3}$ and Sabine Mondié ${ }^{1}$ \\ ${ }^{1}$ Department of Atomatic Control, CINVESTAV-IPN, Mexico D.F., Mexico \\ Email: smondie@ctrl.cinvestav.mx, msaldivar@ctrl.cinvestav.mx \\ ${ }^{2}$ Institut de Recherche en Communications et Cybernétique de Nantes, Nantes, France \\ ${ }^{3}$ Laboratoire de Recherche, Gipsa-lab Grenoble Images Parole Signal Automatique, Grenoble, France \\ Email: Alexandre.Seuret@gipsa-lab.grenoble-inp.fr
}

\begin{abstract}
This paper deals with exponential stabilization of the class of nonlinear neutral type time-delay systems that can be transformed into a multi-model system. The approach is based on Lyapunov-Krasovskii techniques and uses a descriptor representation. The exponential stability properties are proved using an appropriate change of variables associated with a polytopic representation. The results are given in terms of LMIs. As an application example, we determine an effective stabilizing controller for an oilwell drilling system.
\end{abstract}

Keywords: Neutral type time-delay systems, nonlinear systems, polytopic representation, exponential stabilization.

\section{INTRODUCTION}

It is well known that nonlinear systems are indeed models closer to reality in the sense that their validity is not necessarily limited to an immediate neighborhood of an operating point or a reference trajectory.

In [1] the author presents stabilizability results for nonlinear retarded type time-delay systems which can be represented in two different ways: as multi-model systems and as uncertain systems.

We are interested in the stabilization of nonlinear neutral type time-delay systems which can be transformed into a multi-model system, i.e., a set of linear models nonlinearly weighted. A multi-model neutral type system can be represented as follows

$$
\begin{aligned}
\dot{x}(t)-D \dot{x}\left(t-\tau_{1}\right)= & \sum_{\substack{i \in I^{r}\\
}} h_{i}\left(x_{t}\right)\left\{A_{i} x(t)+A_{i \tau_{1}} x\left(t-\tau_{1}\right)\right. \\
& \left.+B_{\tau_{0}} u\left(t-\tau_{0}\right)\right\}
\end{aligned}
$$

*This work was supported by CONACYT under grant 61076 and scholarship 209927. where the set $I^{r}$ is the set of integers $\{1, \ldots, r\}, r$ is the number of subsystems required to describe the multimodel system. The functions $h_{i}(\cdot)$ are scalar weighting functions satisfying the convexity conditions:

$$
\sum_{i \in I^{r}} h_{i}\left(x_{t}\right)=1 \quad \forall i=1, \ldots, r, \quad h_{i}\left(x_{t}\right) \geq 0 .
$$

The proposal of a Lyapunov-Krasovskii functional and a descriptor representation of the neutral type time-delay system allow us to find a stabilizing controller for this particular kind of nonlinear systems through the solution of linear matrix inequalities.

Our motivation is the exponential stabilitzation of an oilwell drilling system. Oilwell drillstrings are mechanisms that play a key role in the petroleum extraction industry. These devices are complex dynamic systems with many unknown and varying parameters due to the fact that drillstring characteristics change as the drilling operation makes progress. The drilling system is described by an hyperbolic partial differential equation with mixed boundary conditions. Through the D'Alembert method this model can be easily transformed into a neutral type delay system which describes the behavior of the system at the ground level. The torque on the bit is described by a nonlinear function which depends on the angular velocity at the bottom extremity. Under an appropriate change of variables we can obtain a polytopic representation of the drilling system.

The paper is organized as follows: In Section II we present the distributed parameter model describing the drilling system and the nonlinear equivalent neutral type delay model obtained trough the D'Alembert transformation. In section III we present the multi-model approximation of the drilling nonlinear system. Section IV concerns the $\alpha$-stability analysis of the open loop multimodel system, then, we determine the LMI conditions for the exponential stability of the closed loop system. The synthesis of the controller gain is obtained. In Section V 
we present the numerical analysis of the drilling system. Conclusions are presented in the last section.

\section{NONLINEAR MODEL OF THE DRILLING SYSTEM}

The main process during well drilling for oil is the creation of borehole by a rock-cutting tool called bit. The drillstring consists of the BHA (bottom hole assembly) and drillpipes screwed end to end to each other to form a long pipe. The BHA comprises the bit, stabilizers (at least two spaced apart) which prevent the drillstring from balancing, and a series of pipe sections which are relatively heavy known as drill collars. While the length of the BHA remains constant, the total length of the drill pipes increases as the borehole depth does. An important element of the process is the drilling mud or fluid which among others, has the function of cleaning, cooling and lubricating the bit. The drillstring is rotated from the surface by an electrical motor. The rotating mechanism can be of two types: a rotary table or a top drive.

The drill pipe is considered as a beam in torsion. A lumped inertia $I_{B}$ is chosen to represent the assembly at the bottom hole and a damping $\beta \geq 0$ which includes the viscous and structural damping, is assumed along the structure. The drillstring is rotated from the surface $(\xi=$ 0 ) by an electrical motor, $\Omega$ is the angular velocity coming from the rotor that does not match the rotational speed of the load $\frac{\partial \theta}{\partial t}(0, t)$. This sliding speed results in the local torsion of the drillstring. The other extremity $(\xi=L)$, is subject to a torque $T$, which is a function of the bit speed. The mechanical system is described by the following partial differential equation:

$$
\begin{gathered}
G J \frac{\partial^{2} \theta}{\partial \xi^{2}}(\xi, t)-I \frac{\partial^{2} \theta}{\partial t^{2}}(\xi, t)-\beta \frac{\partial \theta}{\partial t}(\xi, t)=0 \\
\xi \in(0, L), t>0
\end{gathered}
$$

with boundary conditions

$$
\begin{aligned}
G J \frac{\partial \theta}{\partial \xi}(0, t) & =c_{a}\left(\frac{\partial \theta}{\partial t}(0, t)-\Omega(t)\right) ; \\
G J \frac{\partial \theta}{\partial \xi}(L, t)+I_{B} \frac{\partial^{2} \theta}{\partial t^{2}}(L, t) & =-T\left(\frac{\partial \theta}{\partial t}(L, t)\right),
\end{aligned}
$$

where $\theta(\xi, t)$ is the angle of rotation, $I$ is the inertia, $G$ is the shear modulus and $J$ is the geometrical moment of inertia.

Considering that the damping $\beta$ is negligible, the distributed parameter model (3) reduces to the unidimensional wave equation. Using the D'Alembert transformation we can describe the drilling behavior with the following neutral type delay equation:

$$
\begin{gathered}
\ddot{w}(t)-\Upsilon \ddot{w}(t-2 \Gamma)+\Psi \dot{w}(t)+\Psi \Upsilon \dot{w}(t-2 \Gamma)= \\
-\frac{1}{I_{B}} T(\dot{w}(t))+\frac{1}{I_{B}} \Upsilon T(\dot{w}(t-2 \Gamma)) \\
+\Pi \Omega(t-\Gamma),
\end{gathered}
$$

where $\dot{w}(t)$ is the angular velocity at the bottom extremity, and $\Upsilon=\frac{c_{a}-\sqrt{I G J}}{c_{a}+\sqrt{I G J}}, \Psi=\frac{\sqrt{I G J}}{I_{B}}, \Gamma=\sqrt{\frac{I}{G J}} L, \Pi=\frac{2 \Psi c_{a}}{c_{a}+\sqrt{I G J}}$.

For the details of the transformation the reader is referred to [3], [4].

The drillstring interaction with the borehole gives rise to a wide variety of non-desired oscillations which are classified depending on the direction they appear. Three main types of vibrations can be distinguished: torsional (stick-slip oscillations), axial (bit bouncing phenomenon) and lateral (whirl motion due the out-of-balance of the drillstring). Torsional drillstring vibrations appear due to downhole conditions, such as significant drag, tight hole, and formation characteristics. It can cause the bit to stall in the formation while the rotary table continues to rotate. When the trapped torsional energy (similar to a woundup spring) reaches a level that the bit can no longer resist, the bit suddenly comes loose, rotating and whipping at very high speeds. This stick-slip behavior can generate a torsional wave that travels up the drillstring to the rotary top system. Because of the high inertia of the rotary table, it acts like a fixed end to the drillstring and reflects the torsional wave back down the drillstring to the bit. The bit may stall again, and the torsional wave cycle repeats as explained in [6]. The whipping and high speed rotations of the bit in the slip phase can generate both severe axial and lateral vibrations at the bottom-hole assembly. The vibrations can originate problems such as drill pipe fatigue problems, drillstring components failures, wellbore instability. They contribute to drillpipe fatigue and are detrimental to bit life.

The following nonlinear equation introduced in [5] approximates the physical phenomenon at the bottom hole

$$
T(\dot{w}(t))=c_{b} \dot{w}(t)+W_{o b} R_{b} \mu_{s b} e^{-\frac{\gamma_{b}}{v_{f}} \dot{w}(t)} \operatorname{sgn}(\dot{w}(t)) .
$$

The term $c_{b} \dot{w}(t)$ is a viscous damping torque at the bit which approximates the influence of the mud drilling and the term $W_{o b} R_{b} \mu_{s b} e^{-\frac{\gamma_{b}}{v_{f}} \dot{w}(t)} \operatorname{sgn}(\dot{w}(t))$ is a dry friction torque modelling the bit-rock contact. $R_{b}>0$ is the bit radius, $W_{o b}>0$ the weight on the bit, $\mu_{s b} \in(0,1)$ is the static friction coefficient and $0<\gamma_{b}<1$ is a constant defining the velocity decrease rate. The constant velocity $v_{f}>0$ is introduced in order to have appropriate units.

The friction torque (5) leads to a decreasing torque-onbit with increasing bit angular velocity for low velocities which acts as a negative damping (Stribeck effect) and is the cause of stick-slip self-excited vibrations. The exponential decaying behavior of $T$ coincides with experimental torque values.

With the introduction of the torque on the bit model we obtain the following nonlinear expression to describe 
the drilling behavior at the ground level:

$$
\begin{gathered}
\ddot{w}(t)-\Upsilon \ddot{w}(t-2 \Gamma)+\left(\Psi+\frac{c_{b}}{I_{B}}\right) \dot{w}(t) \\
+\Upsilon\left(\Psi-\frac{c_{b}}{I_{B}}\right) \dot{w}(t-2 \Gamma)=-c_{2} e^{-\frac{\gamma_{b}}{v_{f}} \dot{w}(t)} \operatorname{sgn}(\dot{w}(t)) \\
+\Upsilon c_{2} e^{-\frac{\gamma_{b}}{v_{f}} \dot{w}(t-2 \Gamma)} \operatorname{sgn}(\dot{w}(t-2 \Gamma))+\Pi \Omega(t-\Gamma)
\end{gathered}
$$

where $c_{2}=\frac{W_{o b} R_{b}\left(\mu_{s b}-\mu_{c b}\right)}{I_{B}}$.

\section{Multi-model APproximation of the NONLINEAR SYSTEM}

Consider a nonlinear control system of the form

$$
\begin{aligned}
\dot{x}(t)-D \dot{x}\left(t-\tau_{1}\right)= & f\left(t, x_{t}\right)+g\left(t, x_{t}\right) u(t) \\
& +h\left(t, x_{t}\right) u\left(t-\tau_{0}\right) \\
x(t)= & \phi(t) \quad \forall t \in\left[-\tau_{1}, 0\right]
\end{aligned}
$$

where $f \in \mathbb{R}^{n}$ is a real-valued functional which depends on $t$ and the function $x_{t}, x_{t}(\theta)=x(t+\theta), \theta \in\left[-\tau_{1}, 0\right]$, the functionals $f$ and $g$ take values in $\mathbb{R}^{n \times m}$, the function $\phi(t)$ stands for the initial condition, it is defined on $\left[-\tau_{1}, 0\right]$.

In order to simplify the analysis, we can transform the nonlinear initial system (7) into a multi-model system described by (1).

We choose the following change of variables to represent the nonlinear model of the drilling system (6) as a multimodel system:

$$
\left\{\begin{array}{l}
x_{1}(t)=w(t) \\
x_{2}(t)=\dot{w}(t) \\
x_{3}(t)=e^{-\frac{\gamma_{b}}{v_{f}} x_{2}(t)}
\end{array}\right.
$$

therefore,

$$
\left\{\begin{array}{l}
\dot{x}_{1}(t)=\dot{w}(t) \\
\dot{x}_{2}(t)=\ddot{w}(t) \\
\dot{x}_{3}(t)=-\frac{\gamma_{b}}{v_{f}} \dot{x}_{2}(t) e^{-\frac{\gamma_{b}}{v_{f}} x_{2}(t)}=-\frac{\gamma_{b}}{v_{f}} \dot{x}_{2}(t) x_{3}(t) .
\end{array}\right.
$$

System (6) can be written as

$\dot{x}(t)-D \dot{x}\left(t-\tau_{1}\right)=A(x) x(t)+A_{\tau_{1}}(x) x\left(t-\tau_{1}\right)+B_{\tau_{0}} u\left(t-\tau_{0}\right)$

where $\tau_{0}=\Gamma, \tau_{1}=2 \Gamma, u(t)=\Omega$,

$$
\begin{aligned}
& x=\left[\begin{array}{lll}
x_{1} & x_{2} & x_{3}
\end{array}\right]^{T} \\
& D=\left(\begin{array}{ccc}
0 & 0 & 0 \\
0 & \Upsilon & 0 \\
0 & 0 & 0
\end{array}\right), \quad B_{\tau_{0}}=\left(\begin{array}{c}
0 \\
\Pi \\
0
\end{array}\right) \text {, } \\
& A_{\tau_{1}}(x)=\left(\begin{array}{ccc}
0 & 0 & 0 \\
0 & \Upsilon\left(\frac{c_{b}}{I_{B}}-\Psi\right) & c_{2} \Upsilon \operatorname{sgn}\left(x_{2}\left(t-\tau_{1}\right)\right) \\
0 & 0 & 0
\end{array}\right), \\
& A(x)=\left(\begin{array}{ccc}
0 & 1 & 0 \\
0 & -\left(\Psi+\frac{c_{b}}{I_{B}}\right) & -c_{2} \operatorname{sgn}\left(x_{2}(t)\right) \\
0 & 0 & -\frac{\gamma_{b}}{v_{f}} \dot{x}_{2}(t)
\end{array}\right) \text {. }
\end{aligned}
$$

Notice that the entries of the matrices $D, B_{\tau_{0}}$ are constant, and the entry $c_{2} \Upsilon \operatorname{sgn}\left(x_{2}\left(t-\tau_{1}\right)\right)$ of the matrix $A_{\tau_{1}}(x)$ is bounded. If we consider that $\dot{x}_{2}(t)$ is a bounded variable then, so is the matrix $A(x)$.

In this case, we can obtain a polytopic representation of the matrices $A(x), A_{\tau_{1}}(x)$ as:

$$
\begin{aligned}
& A(x) x(t)+A_{\tau_{1}}(x) x\left(t-\tau_{1}\right)= \\
& \sum_{i \in I^{r}} h_{i}\left(x_{t}\right)\left(A_{i} x(t)+A_{i \tau_{1}} x\left(t-\tau_{1}\right)\right)
\end{aligned}
$$

where $A_{i}, A_{i \tau_{1}}$ have only constant coefficients [1]. The functions $h_{i}\left(x_{t}\right), i \in I^{r}$ are scalar not necessarily known weighting functions satisfying the convexity property (2).

The non-linear drilling system (6) can be written in the polytopic form (1) .

\section{MAIN RESULT}

Firstly, we are going to analyze the $\alpha$-stability of the open loop system, i.e., the system:

$$
\dot{x}(t)-D \dot{x}\left(t-\tau_{1}\right)=\sum_{i \in I^{r}} h_{i}\left(x_{t}\right)\left\{A_{i} x(t)+A_{i \tau_{1}} x\left(t-\tau_{1}\right)\right\} .
$$

To guarantee that the difference operator is stable we assume $|D|<1$.

The change of variable $x_{\alpha}(t)=e^{\alpha t} x(t)$ transforms the system (10) into:

$$
\begin{gathered}
\dot{x}_{\alpha}(t)-D e^{\alpha \tau_{1}} \dot{x}_{\alpha}\left(t-\tau_{1}\right)=\sum_{i \in I^{r}} h_{i}\left(x_{t}\right)\left\{\left(A_{i}+\alpha I_{n}\right) x_{\alpha}(t)\right. \\
\left.+e^{\alpha \tau_{1}}\left(A_{i \tau_{1}}-\alpha D\right) x_{\alpha}\left(t-\tau_{1}\right)\right\}
\end{gathered}
$$

The proposal is to find conditions for which the solution $x_{\alpha}=0$ of the transformed system (11) is asymptotically stable. Clearly, these conditions will assure the exponential stability of the original system (10).

Theorem 1: The solution $x(t)=0$ of the system (10) is $\alpha$-stable if there exist matrices $0<P_{1}=P_{1}^{T}, P_{2}, P_{3}$, $Q=Q^{T}$ and $R=R^{T}$, such that for all $i \in I^{r}$ the following linear matrix inequality (LMI) is satisfied

$$
\left(\begin{array}{ccc}
\Psi_{i} & P^{T}\left(\begin{array}{c}
0 \\
e^{\alpha \tau_{1}}\left(A_{i \tau_{1}}-\alpha D\right)
\end{array}\right) & P^{T}\left(\begin{array}{c}
0 \\
e^{\alpha \tau_{1}} D
\end{array}\right) \\
* & -R / \tau_{1} & 0 \\
* & * & -Q
\end{array}\right)<0
$$

where

$$
\begin{aligned}
P: & =\left(\begin{array}{cc}
P_{1} & 0 \\
P_{2} & P_{3}
\end{array}\right), \quad P_{1}=P_{1}^{T}>0 \\
\Psi_{i}: & =P^{T}\left(\begin{array}{cc}
0 & I_{n} \\
\Lambda_{i} & -I_{n}
\end{array}\right)+\left(\begin{array}{cc}
0 & I_{n} \\
\Lambda_{i} & -I_{n}
\end{array}\right)^{T} P \\
& +\left(\begin{array}{cc}
0 & 0 \\
0 & \tau_{1} R+Q
\end{array}\right) \\
\Lambda_{i}: & =A_{i}+\alpha I_{n}+e^{\alpha \tau_{1}}\left(A_{i \tau_{1}}-\alpha D\right)
\end{aligned}
$$


Proof: According to the Leibniz formula,

$$
x_{\alpha}\left(t-\tau_{1}\right)=x_{\alpha}(t)-\int_{t-\tau_{1}}^{t} \dot{x}_{\alpha}(s) d s,
$$

then, we can write the system (11) as

$$
\begin{gathered}
\dot{x}_{\alpha}(t)-D e^{\alpha \tau_{1}} \dot{x}_{\alpha}\left(t-\tau_{1}\right)= \\
\sum_{i \in I^{r}} h_{i}\left(x_{t}\right)\left\{\left(A_{i}+\alpha I_{n}+e^{\alpha \tau_{1}}\left(A_{i \tau_{1}}-\alpha D\right)\right) x_{\alpha}(t)\right. \\
\left.-e^{\alpha \tau_{1}}\left(A_{i \tau_{1}}-\alpha D\right) \int_{t-\tau_{1}}^{t} \dot{x}_{\alpha}(s) d s\right\} .
\end{gathered}
$$

Using the descriptor form introduced in [2] we have

$$
\begin{aligned}
\dot{x}_{\alpha}(t)= & y(t) \\
y(t)= & \sum_{i \in I^{r}} h_{i}\left(x_{t}\right)\left\{D e^{\alpha \tau_{1}} y\left(t-\tau_{1}\right)+\Lambda_{i} x_{\alpha}(t)\right. \\
& \left.-e^{\alpha \tau_{1}}\left(A_{i \tau_{1}}-\alpha D\right) \int_{t-\tau_{1}}^{t} y(s) d s\right\},
\end{aligned}
$$

where

$$
\Lambda_{i}:=A_{i}+\alpha I_{n}+e^{\alpha \tau_{1}}\left(A_{i \tau_{1}}-\alpha D\right)
$$

then, we can write

$$
E\left(\begin{array}{c}
\dot{x}_{\alpha}(t) \\
\dot{y}(t)
\end{array}\right)=\left(\begin{array}{c}
y(t) \\
\sum_{i \in I^{r}} h_{i}\left(x_{t}\right) \cdot \lambda
\end{array}\right)
$$

where $E=\operatorname{diag}\left\{I_{n}, 0\right\}, \lambda=-y(t)+D e^{\alpha \tau_{1}} y\left(t-\tau_{1}\right)+$ $\Lambda_{i} x_{\alpha}(t)-e^{\alpha \tau_{1}}\left(A_{i \tau_{1}}-\alpha D\right) \int_{t-\tau_{1}}^{t} y(s) d s$.

Following [2], we use the Lyapunov-Krasovskii functional

$$
\begin{gathered}
V_{\alpha}(t)=\left(\begin{array}{cc}
x_{\alpha}^{T}(t) & y^{T}(t)
\end{array}\right) \operatorname{EP}\left(\begin{array}{c}
x_{\alpha}(t) \\
y(t)
\end{array}\right) \\
+\int_{-\tau_{1}}^{0} \int_{t+\theta}^{t} y^{T}(s) R y(s) d s d \theta+\int_{t-\tau_{1}}^{t} y^{T}(s) Q y(s) d s \\
P=\left(\begin{array}{cc}
P_{1} & 0 \\
P_{2} & P_{3}
\end{array}\right), P_{1}=P_{1}^{T}>0, R>0, Q>0 .
\end{gathered}
$$

The functional $V_{\alpha}(t)$ is positive definite since

$$
\left(\begin{array}{cc}
x_{\alpha}^{T}(t) & y^{T}(t)
\end{array}\right) E P\left(\begin{array}{c}
x_{\alpha}(t) \\
y(t)
\end{array}\right)=x_{\alpha}^{T}(t) P_{1} x_{\alpha}(t) .
$$

Notice that $E P=P^{T} E$, taking the derivative in $t$ of $V_{\alpha}(t)$ we obtain

$$
\begin{aligned}
\dot{V}_{\alpha}(t) & =2\left(\begin{array}{cc}
x_{\alpha}^{T}(t) & y^{T}(t)
\end{array}\right) P^{T}\left(\begin{array}{c}
y(t) \\
\sum_{i \in I^{r}} h_{i}\left(x_{t}\right) \cdot \lambda
\end{array}\right) \\
& +\tau_{1} y^{T}(t) R y(t)-\int_{t-\tau_{1}}^{t} y^{T}(s) R y(s) d s \\
& +y^{T}(t) Q y(t)-y^{T}\left(t-\tau_{1}\right) Q y\left(t-\tau_{1}\right) .
\end{aligned}
$$

Setting $\xi=\left(\begin{array}{lll}x_{\alpha}(t) & y(t) & y\left(t-\tau_{1}\right.\end{array}\right)$ we can write

$$
\begin{aligned}
\dot{V}_{\alpha}(t)= & \xi^{T}\left(\begin{array}{cc}
\tilde{\Psi}_{i} & P^{T}\left(\begin{array}{c}
0 \\
e^{\alpha \tau_{1}} D
\end{array}\right) \\
\left(\begin{array}{cc}
0 & \left.e^{\alpha \tau_{1}} D^{T}\right) P
\end{array}\right. & -Q
\end{array}\right) \xi \\
& +\eta-\int_{t-\tau_{1}}^{t} y^{T}(s) R y(s) d s
\end{aligned}
$$

where

$$
\begin{aligned}
& \eta=-2 \int_{t-\tau_{1}}^{t}\left(x_{\alpha}^{T}(t) y^{T}(t)\right) \cdot P^{T} . \\
& \cdot\left(\begin{array}{c}
0 \\
e^{\alpha \tau_{1}}\left(A_{i \tau_{1}}-\alpha D\right)
\end{array}\right) y(s) d s, \\
& \tilde{\Psi}_{i} \quad: \quad=\sum_{i \in I^{r}} h_{i}\left(x_{t}\right)\left\{P^{T}\left(\begin{array}{cc}
0 & I_{n} \\
\Lambda_{i} & -I_{n}
\end{array}\right)\right. \\
& \left.+\left(\begin{array}{cc}
0 & I_{n} \\
\Lambda_{i} & -I_{n}
\end{array}\right)^{T} P+\left(\begin{array}{cc}
0 & 0 \\
0 & \tau_{1} R+Q
\end{array}\right)\right\} \text {. }
\end{aligned}
$$

In order to obtain an upper bound on $\eta$, we use the following property.

For all vectors $a, b \in \mathbb{R}^{n}$ and positive definite matrix $\mathbb{R}^{n \times n}$, the following inequality is satisfied

$$
\pm 2 a^{T} b \leq a^{T} R^{-1} a+b^{T} R b .
$$

Then, we have that

$$
\begin{aligned}
\eta \leq & \left(x_{\alpha}^{T}(t) y^{T}(t)\right) \cdot P^{T} \\
& \cdot\left(\begin{array}{c}
0 \\
e^{\alpha \tau_{1}}
\end{array}\left(\begin{array}{c}
\left.A_{i \tau_{1}}-\alpha D\right)
\end{array}\right) \cdot \tau_{1} R^{-1} \cdot\right. \\
& \left(\begin{array}{cc}
0 & e^{\alpha \tau_{1}}\left(A_{i \tau_{1}}-\alpha D\right)^{T}
\end{array}\right) \cdot P \cdot\left(\begin{array}{c}
x_{\alpha}(t) \\
y(t)
\end{array}\right) \\
& +\int_{t-\tau_{1}}^{t} y^{T}(s) R y(s) d s
\end{aligned}
$$

From (13) and (14),

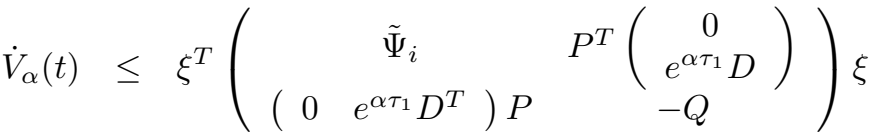

$$
\begin{aligned}
& +\left(\begin{array}{ll}
x_{\alpha}^{T}(t) & y^{T}(t)
\end{array}\right) \cdot P^{T} . \\
& \cdot\left(\begin{array}{c}
0 \\
e^{\alpha \tau_{1}}\left(A_{i \tau_{1}}-\alpha D\right)
\end{array}\right) \cdot\left(\tau_{1} R^{-1}\right) \cdot \\
& \left(\begin{array}{cc}
0 & e^{\alpha \tau_{1}}\left(A_{i \tau_{1}}-\alpha D\right)^{T}
\end{array}\right) \cdot P \cdot\left(\begin{array}{c}
x_{\alpha}(t) \\
y(t)
\end{array}\right)
\end{aligned}
$$

Finally, using Schur complements, the system (10) is asymptotically stable if every matrix, $i \in I^{r}$

$$
\left(\begin{array}{ccc}
\Psi_{i} & P^{T}\left(\begin{array}{c}
0 \\
e^{\alpha \tau_{1}}\left(A_{i \tau_{1}}-\alpha D\right)
\end{array}\right) & P^{T}\left(\begin{array}{c}
0 \\
e^{\alpha \tau_{1}} D
\end{array}\right) \\
* & -R / \tau_{1} & 0 \\
* & * & -Q
\end{array}\right),
$$

is negative definite, i.e., if the LMI condition (12) is satisfied.

Having determined the criteria for exponential stability for the open loop system (10), the next step is to define an algorithm that allows the synthesis of a gain $K$ such that the feedback control law

$$
u\left(t-\tau_{0}\right)=K x\left(t-\tau_{1}\right) .
$$


exponentially stabilizes the closed loop system

$$
\begin{aligned}
\dot{x}(t)-D \dot{x}\left(t-\tau_{1}\right)= & \sum_{i \in I^{r}} h_{i}\left(x_{t}\right)\left\{A_{i} x(t)\right. \\
& \left.+\left(A_{i \tau_{1}}+B_{\tau_{0}} K\right) x\left(t-\tau_{1}\right)\right\}
\end{aligned}
$$

with a guaranteed rate of convergence $\alpha$.

Replacing the matrix $A_{i \tau_{1}}$ by the matrix $A_{i \tau_{1}}+B_{\tau_{0}} K$ in Theorem 1, that the solution $x(t)=0$ of the system (16) is $\alpha$-stable if there exist matrices $0<P_{1}=P_{1}^{T}, P_{2}, P_{3}$, $Q=Q^{T}, R=R^{T}$ such that for all $i \in I^{r}$ the following bilinear matrix inequality is satisfied

$$
\left(\begin{array}{ccc}
\Psi_{i} & P^{T}\left(\begin{array}{c}
0 \\
e^{\alpha \tau_{1}} \chi
\end{array}\right) & P^{T}\left(\begin{array}{c}
0 \\
e^{\alpha \tau_{1}} D
\end{array}\right) \\
* & -R / \tau_{1} & 0 \\
* & * & -Q
\end{array}\right)<0
$$

where

$$
\begin{aligned}
P: & =\left(\begin{array}{cc}
P_{1} & 0 \\
P_{2} & P_{3}
\end{array}\right), \quad P_{1}=P_{1}^{T}>0, \\
\Psi_{i}: & =P^{T}\left(\begin{array}{cc}
0 & I_{n} \\
\Lambda_{i} & -I_{n}
\end{array}\right)+\left(\begin{array}{cc}
0 & I_{n} \\
\Lambda_{i} & -I_{n}
\end{array}\right)^{T} P \\
& +\left(\begin{array}{cc}
0 & 0 \\
0 & \tau_{1} R+Q
\end{array}\right) \\
\Lambda_{i}: & =A_{i}+\alpha I_{n}+e^{\alpha \tau_{1}}\left(A_{i \tau_{1}}+B_{\tau_{0}} K-\alpha D\right), \\
\chi: & =\left(A_{i \tau_{1}}+B_{\tau_{0}} K-\alpha D\right) .
\end{aligned}
$$

A well known synthesis gain technique which overcome the bilinearity of the conditions was introduced by [7]. It consists in to set

$$
P_{3}=\epsilon P_{2}, \quad \epsilon \in \mathbb{R}
$$

where $P_{2}$ is a nonsingular matrix, and

$$
\bar{P}=P_{2}^{-1} .
$$

Define $\bar{P}_{1}=\bar{P}^{T} P_{1} \bar{P}, \bar{R}=\bar{P}^{T} R \bar{P}$, and $Y=K \bar{P}$. Multiplying the right side of (17) by $\Delta_{3}=\operatorname{diag}\{\bar{P}, \bar{P}, \bar{P}\}$ and the left side by $\Delta_{3}^{T}$, we obtain the LMI stabilization condition stated in the following theorem.

Theorem 2: The system (16) is $\alpha$-stabilizable if there exist a real number $\epsilon>0$ and $n \times n$ matrices $\bar{P}_{1}>0, \bar{P}$, $\bar{Q}=\bar{Q}^{T}, \bar{R}=\bar{R}^{T}$, and $Y$ such that for all $i \in I^{r}$ the following linear matrix inequality (LMI) is satisfied

$$
\left(\begin{array}{ccc}
\Phi_{i} & \left(\begin{array}{c}
e^{\alpha \tau_{1}} \vartheta \\
\epsilon e^{\alpha \tau_{1}} \vartheta
\end{array}\right) & \left(\begin{array}{c}
e^{\alpha \tau_{1}} D \bar{P} \\
\epsilon e^{\alpha \tau_{1}} D \bar{P}
\end{array}\right) \\
* & -\bar{R} / \tau_{1} & 0 \\
* & * & -\bar{Q}
\end{array}\right)<0
$$

where

$$
\begin{aligned}
\vartheta & :=\left(A_{i \tau_{1}}-\alpha D\right) \bar{P}+B_{\tau_{0}} Y \\
\Phi_{i} & =\left(\begin{array}{cc}
\Phi_{11} & \Phi_{12} \\
* & \Phi_{22}
\end{array}\right)
\end{aligned}
$$

$$
\begin{aligned}
\Phi_{11}= & \left(A_{i}+\alpha I_{n}+e^{\alpha \tau_{1}}\left(A_{i \tau_{1}}-\alpha D\right)\right) \bar{P} \\
& +\bar{P}^{T}\left(A_{i}+\alpha I_{n}+e^{\alpha \tau_{1}}\left(A_{i \tau_{1}}-\alpha D\right)\right)^{T} \\
& +B_{\tau_{0}} Y+Y^{T} B_{\tau_{0}}^{T} \\
\Phi_{12}= & \bar{P}_{1}^{T}-\bar{P}+\epsilon \bar{P}^{T}\left(A_{i}+\alpha I_{n}+e^{\alpha \tau_{1}}\left(A_{i \tau_{1}}-\alpha D\right)\right)^{T} \\
& +\epsilon Y^{T} B_{\tau_{0}}^{T} \\
\Phi_{22}= & -\epsilon\left(\bar{P}+\bar{P}^{T}\right)+\tau_{1} \bar{R}+\bar{Q} .
\end{aligned}
$$

Moreover, the feedback gain is given by

$$
K=Y \bar{P}^{-1} .
$$

\section{NUMERICAL RESUlT}

Now, we are able to find a stabilizing control law for the oilwell drilling system using the results of Section IV.

The model parameters used in the sequel are:

$$
\begin{array}{ccc}
G=79.3 \times 10^{9} \mathrm{~N} / \mathrm{m}^{2}, & I=0.095 \mathrm{Kg} \cdot \mathrm{m}, & L=1172 \mathrm{~m}, \\
J=1.19 \times 10^{-5} \mathrm{~m}^{4} & R b=0.155575, & v_{f}=1, \\
W_{o b}=97347 \mathrm{~N}, & I_{B}=89 \mathrm{Kgm}^{2} & c_{a}=2000 \mathrm{Nms}, \\
c_{b}=0.03 \mathrm{Nms} / \mathrm{rad}, & \mu_{s b}=0.8, & \gamma_{b}=0.9 \\
v_{\text {ref }}=20 \mathrm{rad} / \mathrm{s} & D e c_{\max }=-50 & A c c_{\max }=50
\end{array}
$$

and the simulations are performed using the variable step Matlab-Simulink solver ode45 (Dormand Prince Method).

Using the above parameters, the matrices $A(x), A_{\tau_{1}}(x)$, $B_{\tau_{0}}$ and $D$ of the oilwell drilling model (8) take the following values:

$$
\begin{aligned}
D & =\left(\begin{array}{ccc}
0 & 0 & 0 \\
0 & 0.7396 & 0 \\
0 & 0 & 0
\end{array}\right), B_{\tau_{0}}=\left(\begin{array}{c}
0 \\
5.8523 \\
0
\end{array}\right), \\
A(x) & =\left(\begin{array}{ccc}
0 & 1 & 0 \\
0 & -3.3645 & -136.1327 \operatorname{sgn}\left(x_{2}(t)\right) \\
0 & 0 & -0.9 \dot{x}_{2}(t)
\end{array}\right) \\
A_{\tau_{1}}(x) & =\left(\begin{array}{ccc}
0 & 0 & 0 \\
0 & -2.4878 & 100.6802 \operatorname{sgn}\left(x_{2}\left(t-\tau_{1}\right)\right) \\
0 & 0 & 0
\end{array}\right) .
\end{aligned}
$$

In order to obtain a polytopic representation of the system, $A(x)$ and $A_{\tau_{1}}(x)$ must be bounded functions.

There are three independent functions involved: $\dot{x}_{2}(t)$, $\operatorname{sgn}\left(x_{2}(t)\right)$ and $\operatorname{sgn}\left(x_{2}\left(t-\tau_{1}\right)\right)$.

The variable $\dot{x}_{2}(t)$ represents the angular acceleration at the bottom end of the drillstring, this is clearly a bounded variable in real applications. The variables $\operatorname{sgn}\left(x_{2}(t)\right)$ and $\operatorname{sgn}\left(x_{2}\left(t-\tau_{1}\right)\right)$ take only the values 1 and 0 .

Under the assumption that $A(x)$ and $A_{\tau_{1}}(x)$ are bounded, we can obtain a polytopic representation in the form (9), where $i \in I^{r}=2^{3}=8$.

We can write

$$
A_{i}=\left(\begin{array}{ccc}
0 & 1 & 0 \\
0 & -3.3645 & a_{23}^{i}(x) \\
0 & 0 & a_{33}^{i}(x)
\end{array}\right)
$$


with

$$
\begin{aligned}
-136.1327 & =a_{23}^{1} \leq a_{23}^{i}(x) \leq a_{23}^{2}=0 \\
-0.9 A c c_{\max } & =a_{33}^{1} \leq a_{33}^{i}(x) \leq a_{33}^{2}=-0.9 D e c_{\max }
\end{aligned}
$$

where $A c c_{\max }$ and $D e c_{\max }$ stand for the maximum acceleration and deceleration respectively, and

$$
A_{i \tau_{1}}(x)=\left(\begin{array}{ccc}
0 & 0 & 0 \\
0 & -2.4878 & a_{\tau_{1} 23}^{i}(x) \\
0 & 0 & 0
\end{array}\right)
$$

with

$$
0=a_{\tau_{1} 23}^{1}(x) \leq a_{\tau_{1} 23}^{i}(x) \leq a_{\tau_{1} 23}^{2}(x)=100.6802 .
$$

The simulation results of Figure 1 show the angular velocity at the bottom end of the drillstring $\left(x_{2}(t)\right)$ for $\Omega=20 \mathrm{rad} / \mathrm{s}$.

Applying the result of Theorem 2 to system (1) in closed loop with the control law

$$
u\left(t-\tau_{0}\right)=K x\left(t-\tau_{1}\right),
$$

we obtain feasible results with

$$
K=Y \bar{P}^{-1}=\left(\begin{array}{lll}
0 & 0.44 & -4.25
\end{array}\right) .
$$

Then, the stabilizing control law for the drilling system (6) is given by

$$
\Omega(t)=u(t)=0.44 \dot{w}(t)-4.25 e^{-\frac{\gamma_{b}}{v_{f}} \dot{w}(t)}+v_{\text {ref }} .
$$

The simulation results of Figure 2 show the expected exponential convergence of the variable $\dot{w}(t)\left(x_{2}(t)\right)$ of the system (6) in closed loop with the control law (19) where $v_{r e f}$ is the angular velocity reference.

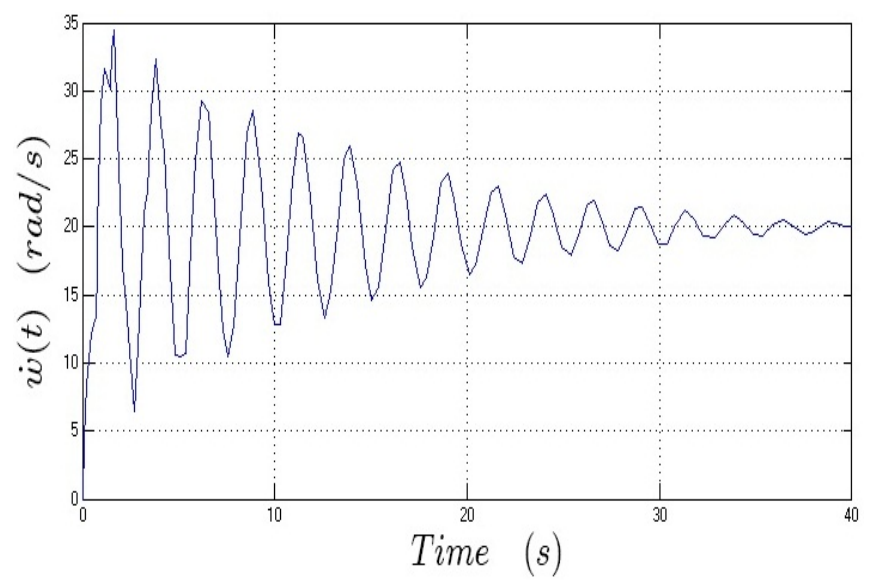

Fig. 1. Simulation of trajectory $\dot{w}(t)$ of the drilling system (6) for $\Omega=20 \mathrm{rad} / \mathrm{s}$.

\section{Conclusion}

The exponential stabilizability of the class of nonlinear neutral type time-delay systems which can be transformed into a multi-model system is investigated in this paper. We have extended the results presented in [1] to the case of neutral type time-delay systems, and we have applied the main result of this paper to the oilwell drilling system.

We have found an effective stabilizing controller which substantially eliminates the oscillations in the angular velocity at the bottom end of the drill string.

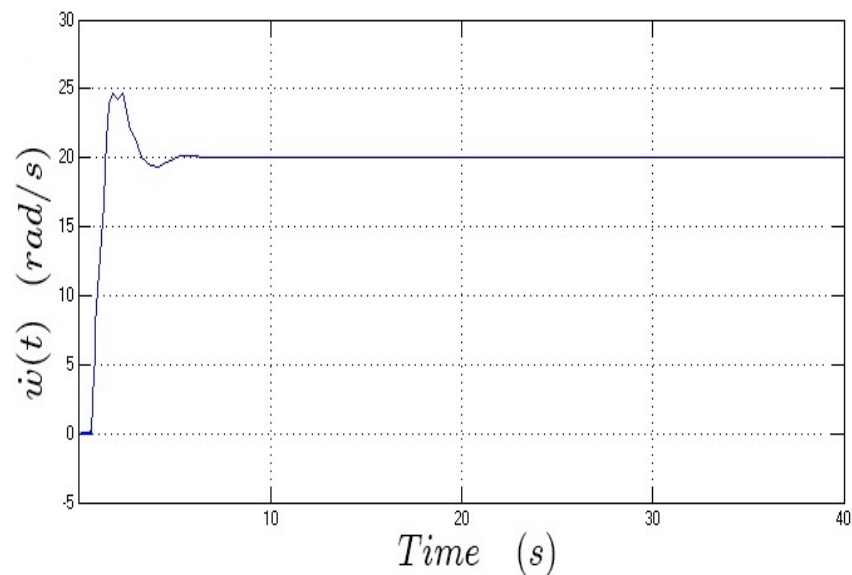

Fig. 2. Simulation of traectory $\dot{w}(t)$ of the drilling system (6) in closed loop with the control law (19).

\section{REFERENCES}

[1] Alexandre Seuret, Commande et observation des systèmes à retard variables, théorie et applications, Thesis $\mathrm{PhD}$, Ecole Centrale de Lille, Univiersité des Sciences et Technologies de Lille, pp. 57-94, 2006.

[2] Emilia Fridman, New Lyapunov-Krasovskii functionals for stability of linear retarded and neutral type systems, Systems \& Control Letters 43, 309-319, 2001.

[3] M. B. Saldivar, S. Mondié, J. J. Loiseau, Reducing stick-slip oscillations in oilwell drillstrings, 6th International Conference on Electrical Engineering, Computing Science and Automatic Control, CCE, pp. 1-6 Toluca, México, 2009.

[4] M. B. Saldivar, S. Mondié, J. J. Loiseau, and V. Rasvan, Stick-slip Oscillations in Oillwell Drilstrings: Distributed Parameter and Neutral Type Retarded Model Approaches, Accepted in IFAC 2011 Milan Italy.

[5] E. Navarro, D. Cortés, Sliding-mode of a multi-DOF oilwell drillstring with stick-slip oscillations, Proceedings of the 2007 American Control Conference. New York City, USA, 2007, pp. 3837-3842.

[6] E. Navarro, R. Suárez, Practical approach to modelling and controlling stick-slip oscillations in oilwell drillstrings, Proceedings of the 2004 IEEE International Conference on Control Applications, pp. 1454-1460.

[7] V. Suplin, E. Fridman and U. Shaked, $\mathrm{H}^{\infty}$ control of linear uncertain time-delay systems - a projection approach. Proceedings of the IEEE Conference on Decision and Control, Bahamas, 2004. 